\title{
Human-Robot Teams Collaborating Socially, Organizationally, and Culturally
}

\author{
Stephen M. Fiore ${ }^{1}$, Norman L. Badler ${ }^{2}$, Lotzi Boloni ${ }^{1}$, Michael A. Goodrich ${ }^{3}$, Annie S. Wu ${ }^{1}$, and Jessie Chen $^{4}$ \\ ${ }^{1}$ University of Central Florida, ${ }^{2}$ University of Pennsylvania, ${ }^{3}$ Brigham Young University, ${ }^{4}$ Army Research Lab
}

We describe an approach examining multi-level collaboration challenges by integrating social, organizational, and cultural factors for human-robot teams operating in the real world. We discuss the research at three levels of social interaction: within a team, within a social environment, and within a culture. We first describe research exploring psychologically and biologically inspired models of behavior to extend the capabilities of heterogeneous multi-human, multi-robot teams. We then discuss research issues that must be addressed to provide insights on how robots can correctly vary actions in response to cultural populations and geospatial environments by recognizing and properly interpreting human configurations, cultural artifacts and behaviors. The goal is to make it possible for robots to function effectively within dynamic operational and social situations.

\section{INTRODUCTION}

Human-robot teams are increasingly interacting, not only in more complex operational environments, but in situations requiring contact and sometimes collaboration with civilians. As such, research is needed which examines collaboration along multiple levels. We describe how to examine this via integration of the social, organizational, and cultural factors required for robots collaborating within teams operating in the real world. Such research is fundamental to making it possible for robots to function effectively within social situations.

We propose a coordinated set of research approaches at three levels of social interaction for human-robot teams: within a team, within a social environment, and within a culture. First, we describe how psychologically and biologically inspired models of behavior can inform team structures and roles and be used to support heterogeneous multi-human, multi-robot teams. Second, we discuss how social-cognitive factors can inform collaboration within teams and with civilians. Third, we provide insights on how robots can correctly vary actions in response to cultural populations and geospatial environments by recognizing and properly interpreting human configurations, cultural artifacts and behaviors.

\section{TEAM AND ORGANIZATIONAL STRUCTURE}

Research in team organizational structures for collaborating agents has successfully employed explicit social rules as an organizing principle. Hierarchically structured teams allow for complex coordination and cooperation when information is available for planning (cf. Crandall et al., 2005; Goodrich et al., 2007; Whetten et al., 2010). Such teams can be agile and responsive if coordination strategies are well designed. The tradeoff for the precision and responsiveness of these teams is that they tend to be more vulnerable to errors, variability, and unanticipated situations. Nevertheless, there is clear evidence that hierarchical teams are effective. In particular, the high levels of coordination in such teams make possible the management of multiple robots simultaneously by a single human. But more is required for coordination to successfully unfold in other settings. For that, research can rely on bioinspired models.

Many biological distributed multi-agent systems exhibit reliable levels of adaptability and robustness, often even under extreme and unexpected conditions. Some of the most interesting and most well studied examples are found in social insect societies. These examples suggest that response thresholds are an effective mechanism for specialization. Variability in response thresholds has been found to lead to stable and effective division of labor in honeybee thermoregulation (Jones et al., 2004). Dynamic reinforcement can further improve system effectiveness by allowing adaptation over time. Ravary et al. (2007) find that positive reinforcement alone can lead to specialization in ants. Ants that are more successful at locating prey are more likely to forage again. Over time, two groups of ants 
(successful and unsuccessful foragers) specialize to form a clear division of labor.

The aforementioned examples from biology tend to be very robust, stable, and adaptable. These systems tend to be massively parallel, with a many to one mapping of agents to roles. These characteristics are particularly compelling considering that these systems are composed of relatively simple agents which interact using very simple, minimal communication (likely nothing as complex as negotiation or reasoning). Importantly, some of these principles have already been successfully transferred to robotic multi-agent systems (Krieger \& Billeter, 2000; Krieger, Billeter, $\&$ Keller, 2000). One of the keys to the ability of these systems to self organize is variability in agent behavior in which agents respond appropriately, but not identically, to similar situations.

\section{Responsive and Robust Organizations}

We suggest that research needs to build upon these approaches in order to build team structures which are both responsive and robust. From this perspective, research must examine how different types and degrees of variation may affect the ability of human-robot systems to self organize and affect the robustness, stability, and adaptability of these systems. In this way, human-robot teams could possess the capability for dynamic role management. Allocation of roles and responsibilities in bio-inspired and hierarchical approaches are, in some respect, two extremes, each with their own benefits. On the one hand, there are well-defined hierarchical systems that are highly responsive and can perform with precision, but may be less tolerant to error. On the other hand, there are massively parallel systems, which we believe are robust and fail gracefully, but may be less responsive in emergency situations. We suggest that research can explore the possible ways in which the advantages of these two approaches may be combined. We next describe feasibility by defining the hierarchical positions from the first approach as the roles that we want agents to self-organize into, and then use the second approach to generate the self-organized system consisting of roles in the hierarchy.

The first broad research challenge is to make bio-inspired models, which are inherently robust, more responsive by applying bio-inspired principles to hierarchical systems. This question attempts to understand and model how robots and humans can use bio-inspired principles to adaptively fill roles in a military hierarchy. Not only must individuals coordinate effectively in their hierarchical roles, individuals must also be able to recognize if a position in the hierarchy is vacant and decide whether or not they are appropriate to fill that role. Thus the distributed decision-making approach described above could be used to increase the robustness of the hierarchical structure and compensate for loss and damage to team members in the defined hierarchy.

Research Issue 1. Research must explore the conditions under which such a dynamically adaptable hierarchical team can effectively be formed and maintained, and study the types of hierarchies that evolve in these teams in comparison to human designed teams.

The second broad research question addresses how to make hierarchical systems more robust. In particular, research must understand and model how we can design hierarchical organizations that facilitate robust and flexible performance for multiple human, multiple robot teams. In currentfan-out models, variability in neglect and interaction times can cause the number of manageable robots to drop dramatically. The result is that operators may have to work most of the time at very low workloads, just in case natural variability causes a workload spike. This leads to responsive but lowperforming teams. We suggest that it is desirable to identify organizations of multiple humans and multiple robots that retain responsiveness in the presence of natural variation.

Research Issue 2. Research must explore organizational structures that flexibly allow multiple humans to manage high-performing teams of robots.

The final broad research question addresses how to apply the theoretical results of the first two research questions so that they are compatible with real human-robot military teams. This requires that research more closely examines current military human-robot team structures.

Research Issue 3. Research must explore team structures and organizations to identify those which are likely to be most useful and relevant to military teams. 


\section{Socio-cognitive Cueing}

Social cues, such as gestures and affective expression, are useful tools for nonverbal communication in human teams, and as humans spontaneously elicit these same behaviors with robots in field settings, gestures and affective expression are expected to be important in human robot teaming (e.g., Zhao \& Badler, 2005). Understanding social cues increases soldier effectiveness by providing more context for robots to understand human intent and, therefore, to decrease the need for explicit instructions from a human. In room clearing for example, the use of gestures (e.g., signaling to stop and cease movement) can be used to maintain stealth, and affective cues (e.g., body movement, facial expression, perspiration) may serve as non-invasive indicators of a given state (e.g., stress) and the degree to which it is experienced.

In this context we suggest that research in human-robot teams must leverage existing frameworks which can support understanding how such cues are related to team interaction. For example, using Kendon's continuum as a framework, gestures can be thought of as a scale of meaning that ranges from gesticulation (e.g., simple motion without meaning to emphasize speech) to sign language (e.g., motion to replace speech), where the addition of meaning increases the cultural specificity of a given message. Additional considerations include the biomorphic expression of social cues in human-robot teams where phenomena such as the "uncanny valley" illustrate that increases in realism can have detrimental effects on emotional expression and social presence.

To accomplish this, research must identify the types of cross-cultural social cues necessary to understand human-robot teams interacting with each other and in varied cultural contexts. Providing humans and robots with these cues would enable an additional means of communication in these teams that should effectively facilitate collaboration and development of shared assessment processes (Arkin et al., 2003). For example, these types of paralinguistic social cues can be used to direct visual attention to areas that are used for non-verbal communication (e.g., hands, face), and help robotic team members determine the meaning behind nonverbal symbols (cf. Byun \& Badler, 2002).

Research Issue 4. Research must identify and classify the gestures and social cues relevant to human-robot teaming and provide culturally applicable classifications and recommendations for their modeling.

The next step for such research would be to understand the role of social-cues in attributions and their relation to collaboration in human-robot teams. In order to identify intent and make inferences about another's actions, we often use particular cues (e.g., body language, gesture). In humanagent/human-robot teams, discovering intent and making inferences will inherently be based upon the type of attributions made about the agent or even by the agent (Fiore, Rehfeld, Jentsch, \& Finkelstein, 2006). We suggest that, for effective human-agent interaction to occur, both the agent and the human must be able to gauge the intent of their teammate (Sycara \& Lewis, 2004). This relies upon both verbal and non-verbal communication which is used to assess intent (cf. Gu \& Badler, 2006). Further, this relies upon appropriate common-ground, that is, "a common language for describing tasks" (Liang, Moreland, \& Argote, 1995 p. 386; see also, Clark \& Wilkes-Gibbs, 1986; Fussel \& Krauss, 1989). Research in this area must identify the appropriate level of communication for the correct intent to be conveyed.

Research Issue 5. Research must examine the relation between social-cognitive cues such as affective expressions and gestures and the development of referential communication in the understanding of intent.

Closely related to understanding intent is the complex cognitive process of making inferences. Specifically, in order to understand intent, it is sometimes necessary for the human and/or the agent to make inferences to fill in a gap in their knowledge. This is a complex component of comprehension that is at the core of understanding text and communication (McKoon \& Ratcliff, 1992). There are numerous forms of inferences that commonly occur (see Graesser, Singer, \& Trabasso, 1994), from causal antecedents (to try to understand what caused something to occur) to emotional reaction (how will this person react). We suggest that these play a critical role in effective collaboration in human robot teams (Arkin et al., 2003) and they are an additional research issue to be addressed. 
Research Issue 6. Research must examine how to identify and accurately incorporate modeled gestures to determine how intent-related decisions should be influenced based on body language cues.

\section{Operating within Social Contexts}

Human actions occur in a context of motivations, opportunities, needs, trusts, and desires. Different cultures have varying norms for how actions are selected (Campbell et al., 2006). Cultural mores affect facial expressions, gestures, body movements, posture, visual orientation, physical contacts, spatial behavior, appearance, and non-verbal vocalizations. Interpretation of behavior requires an understanding of culturally specific action associations and probabilities. Understanding culture makes teams more effective by providing context for robot decision-making that decreases the need for explicit instructions from a soldier and providing context for robots that must interact with civilian bystanders. Cultural institutions (e.g., holidays, prayers, weddings, funerals, or work schedules) and known personal roles (e.g., family, tribe, church, government, military, or service provider) must be modeled and used during robot action selection. We argue that narrow approaches must be replaced by extensible general systems to handle novel team circumstances.

Although speculative, such research would need to focus on creating strategies and models for robot interaction with non-team humans in complex social environments. For example, scenarios could be developed where robots interact with noncombatant co-inhabitants of the robot's local environment. This could involve movement in and around groups of civilians of differing ages, mixes of gender, and multiple cultural backgrounds. Within this context, intents ranging from passive to overtly hostile behavior could be incorporated. This research could utilize software such as the CAROSA crowd simulator (Pelechano et al. 2008). Agents in CAROSA have roles, schedules, and needs and are able to execute actions of several generic types: scheduled, opportunistic, reactive, and stochastic. Underlying CAROSA is a detailed Parameterized Action Representation (PAR) for objects, agents (human or otherwise), actions and events. In this theoretical context, consider now some concrete examples for which cultural features are crucial. A robot engaged in a food distribution task must recognize and observe cultural serving priorities. A robot engaged in a crowd management task must understand personal space, and recognize whether groups are forming as family units or unruly clusters. An observer robot should understand the cultural trappings of a wedding and significance of firearms in the celebration.

Research Issue 7. Research must examine how to best represent cultural knowledge relevant to military teams within robotic systems and what observation, representation, and response activities must be culturally parameterized (e.g., approach distance, friendly and unfriendly gestures and postures, or social significance of eye contact).

Further, three broad aspects of cultural dynamics must be modeled for human-robot teams to effectively interact in social contexts. First, they must understand (in context) the behaviors of observed humans. In this case, behavioral variances or anomalies could be predicates for robot decision making or communications with human team members. Second, they must have a culturallysensitive action selection appropriate to a given mission. Here, the robot's action choices could be tailored to the situation and cultural conditions as much as possible, subject to overall mission goals. Third, they must understand the ways in which different actions affect the perceived values of the participants. In this case, research would need to examine the ways in which an interaction affects the different values such as power, dignity, "face", "propriety" as perceived by different cultures. For example, the same action of the robot might be perceived as friendly in one culture but an insult in another; the immediate participant might have a different interpretation than the witnesses, thus some action might be acceptable in private but not in public. Given these, we present the following research issues:

Research Issue 8. Research must examine how observed human actions can be compared to norms (e.g., through a constructed cultural database) and expectations (e.g., through communication and representation of the current mission or task) provided to the robot.

Research Issue 9. Research must examine how to develop a database of socio-cultural behaviors, standards, and geospatial contexts, framed within an action representation that allows rule-based or probabilistic reasoning bi-directionally from 
observations to interpretation and from behavioral requirements to specific action choice.

Research Issue 10. Research must work to develop an empirical model, in which the cultural costs and benefits of an action are provided by a representation acquired through non-parametric machine-learning techniques from representative examples taken from the socio-cultural behavior database.

\section{CONCLUSION}

To fundamentally change interaction between robots and humans interacting within a team, and within a cultural context, robots need to understand a complex amalgam of organizational, social, and cultural factors. Operationally, the individuals with which the robot is interacting may issue commands to the robots, receive information from robots necessary for their specific tasks, or coordinate activities with the robots (e.g., Schurr et al., 2005; Sofge et al. 2004). But there are also social factors involved in that these missions may require interactions with civilians as well. Understanding teammates during a mission, as well as scenes from a cultural aspect, adds new layers of complexity. The semantic and epistemic aspects of reasoning become more important, as only a very small subset of cultural behaviors can be recognized purely from physical description (cf. Powers \& Kiesler, 2006). As such, we have outlined how to make the initial steps necessary to develop organizational, social, and cultural models that could guide these interactions.

In short, successful interactions within such teams are based on models which identify: (1) Roles within the team structure that mimic and support human team behavior, that is, organizational models; (2) Social cues enacted and interpreted during interaction, that is, social models; and, (3) Manifestation of roles and social-cues within cultural contexts, that is, cultural models. Each of these represents progressively larger spans of behavior that are necessary to support robust and responsive collaboration in team, social, and cultural contexts. Such research is fundamental to making it possible for robots to function effectively within operational and social situations.

\section{REFERENCES}

Arkin, R.C., M. Fujita, T. Takagi, \& R. Hasagawa. (2003). An ethological \& emotional basis for human-robot interaction. Robotics \& Autonomous Systems. 42(3-4):191-201.

Byun, M. \& N. Badler. (2002). FacEMOTE: Qualitative parametric modifiers for facial animations. In Proc. 2002 ACM SIGGRAPH/Eurographics Symposium on Computer Animation, San Antonio, TX, July 2002, pages 65-71.

Campbell, A., A. Wu, K. Garfield, R. Shumaker, S. Luke, \& K. De Jong. (2006). Empirical study on the effects of synthetic social structures on teams of autonomous vehicles. In Proc. IEEE International Conference on Networking, Sensing, \& Control, Fort Lauderdale, FL, April 23-25, 2006.

Clark, H. H., \& Wilkes-Gibbs, D. (1986). Referring as a collaborative process. Cognition, 22, 1-39.

Crandall, J.W., M.A. Goodrich, D.R. Olsen, \& C.W. Nielsen. (2005). Validating Human-Robot Interaction schemes in multi-tasking environments. IEEE Transactions on Systems, Man, \& Cybernetics - Part A: Systems \& Humans. 35 (4):438-449.

Fiore, S. M., Jentsch, F., Rehfeld, S., \& Finkelstein, N. (2005). Situation Awareness and Human-Agent Teams: Integrating Socio-cognitive Theories with the Practice of Human Agent-Teamwork. Proceedings of the 11th International Conference on Human-Computer Interaction.

Fussell, S., \& Krauss, R. (1989). The effects of intended audience on message production and comprehension: Reference in a common ground framework. Journal of Experimental Social Psychology, 25, 203-219.

Goodrich, M.A., T.W. McLain, J.D. \&erson, J. Sun, \& J.W. Crall. (2007). Managing autonomy in robot teams: observations from four experiments. In Proc. ACM SIGCHI/SIGART IEEE RAS Conference on HumanRobot Interaction, March 2007, Arlington, Virginia.

Graesser, A. C., Singer, M., \& Trabasso, T. (1994). Constructing inferences during narrative text comprehension. Psychological Review, 101, 371-395.

Gu, E. \& N. Badler. (2006). Visual attention \& eye gaze during multipartite conversations with distractions. Intelligent Virtual Agents,. Marina del Rey, CA.

Jones, J. C., Myerscough, Graham, S., \& Oldroyd, B. P. (2004). Honey Bee Nest Thermoregulation: Diversity Promotes Stability. Science, 305, 5682, 402-404.

Kendon, A. (1988). How gestures can become like words. Cross-cultural Perspectives in Nonverbal Communication, pages 131-141. Editor F. Poyatos. Toronto, Canada: Hogrefe.

Krieger, M. \& Billeter, J-B, (2000). The call of duty: Selforganise task allocation in a population of up to twelve mobile robots. Robotics and Autonomous Systems, 30, 6584.

Krieger, M.J.B. \& J.-B. Billeter, \& L. Keller. (2000). Ant-like task allocation \& recruitment in cooperative robots. Nature. 406(6799):992-95.

Liang, D., Moreland, R., \& Argote, L. (1995). Group versus individual training and group performance: The mediating factor of transactive memory. Personality \& Social Psychology Bulletin, 21, 384-393.

McKoon, G. \& Ratcliff, R. (1992). Inference during reading. Psychological Review, 99, 440-466.

Pelechano, N., Allbeck, J., \& Badler, N. (2008). Virtual Crowds: Methods, Simulation, and Control. Claypool: San Rafael. 
Powers, A. \& S. Kiesler. (2006). The advisor robot: tracing people's mental model from a robot's physical attributes. In Proc. 2006 ACM Conference on Human-Robot Interaction, pages 218-25. New York: Association for Computing Machinery.

Ravary, F., Lecoutey, E., Kaminski, G., Chaline, N., \& Jaisson, P. (2007). Individual experience alone can generate lasting division of labor in ants. Current Biology 17, 1308-1312.

Schurr, N., J. Marecki, M. Tambe, \& P. Scerri. (2005). Towards flexible coordination of human-agent teams. Int. Jour. of Multiagent \& Grid Systems. 1(1):3-16.

Sofge, D., G. Trafton, N. Cassimatis, D. Perzanowski, M. Bugajska, W. Adams, \& A. Schultz. (2004). Human-robot collaboration \& cognition with an autonomous mobile robot. In Proc. 8th Conference on Intelligent Autonomous Systems (IAS-8), March 2004, pages 80-87.

Sycara, K. \& Lewis, M. (2004). Integrating Intelligent Agents into Human Teams. In E. Salas and S. M. Fiore (Eds.), Team Cognition: Understanding the factors driving process and performance (pp. 203-231). Washington, DC: American Psychological Association.

Velagapudi, P., Prokopyev, O., Sycara, K., \& Scerri, P. (2009). Analyzing the performance of randomized information sharing. In Proceedings of the 8th International
Conference on Autonomous Agents and Multiagent Systems, Budapest, Hungary.

Weidenmuller, A. (2004). The control of nest climate in bumblebee (bombus terrestris) colonies: interindividual variability \& self reinforcement in fanning response. Behavioral Ecology. 15(1):120-28.

Whetten, J. M. Goodrich, M. A., \& Guo, Y. (2010). Beyond robot fan-out: Towards multi-operator supervisory control. In Proceedings of IEEE International Conference on Systems, Man, and Cybernetics, Istanbul.

Zhao, L. \& N. Badler. (2005). Acquiring \& validating motion qualities from live limb gestures. Graphical Models. 67(1):1-16.

\section{ACKNOWLEDGEMENT}

This work was supported by the US Army Research Laboratory (ARL) (100005MC). The views and conclusions contained in this document are those of the authors and should not be interpreted as representing the official policies, either expressed or implied, of ARL or the US Government. The US Government is authorized to reproduce and distribute reprints for Government purposes notwithstanding any copyright notation 